\title{
Characterization of binary string statistics for syntactic landmine detection
}

\author{
Ahmed O. Nasif, Brian L. Mark, and Kenneth J. Hintz \\ Dept. of Electrical and Computer Engineering \\ George Mason University \\ Fairfax, VA 22030, U.S.A.
}

\begin{abstract}
Syntactic landmine detection has been proposed to detect and classify non-metallic landmines using ground penetrating radar (GPR). In this approach, the GPR return is processed to extract characteristic binary strings for landmine and clutter discrimination. In our previous work, we discussed the preprocessing methodology by which the amplitude information of the GPR A-scan signal can be effectively converted into binary strings, which identify the impedance discontinuities in the signal.

In this work, we study the statistical properties of the binary string space. In particular, we develop a Markov chain model to characterize the observed bit sequence of the binary strings. The state is defined as the number of consecutive zeros between two ones in the binarized A-scans. Since the strings are highly sparse (the number of zeros is much greater than the number of ones), defining the state this way leads to fewer number of states compared to the case where each bit is defined as a state. The number of total states is further reduced by quantizing the number of consecutive zeros. In order to identify the correct order of the Markov model, the mean square difference (MSD) between the transition matrices of mine strings and non-mine strings is calculated up to order four using training data. The results show that order one or two maximizes this MSD.

The specification of the transition probabilities of the chain can be used to compute the likelihood of any given string. Such a model can be used to identify characteristic landmine strings during the training phase. These developments on modeling and characterizing the string statistics can potentially be part of a real-time landmine detection algorithm that identifies landmine and clutter in an adaptive fashion.
\end{abstract}

Keywords: Landmine detection, Ground penetrating radar, Syntactic pattern recognition, Binary string, Markov chain

\section{INTRODUCTION}

A high-performance, robust and real-time landmine detection system is essential for successful military and humanitarian demining operations. Despite the efforts of the research community over the last 10-15 years, satisfactory performance in the field has yet to be achieved. Most existing solutions are specialized in terms of the metal content of mines and the applied sensor, and typically employ some form of anomaly detection based on probabilistic modeling and pattern recognition. ${ }^{1-3}$ In recent years, multi-sensor solutions are also being studied for performance improvement. ${ }^{4}$

Our efforts are focused on detecting low metal anti-tank landmines using ground penetrating radar (GPR) with high speed and detection performance. We have proposed the syntactic pattern recognition (SPR) method for landmine detection. ${ }^{5}$ In this approach, the GPR signal is preprocessed in two steps to identify the impedance discontinuity profile of the target. ${ }^{6}$ The first step is inverse filtering, which deconvolves the composite scattered signal due to the target and bandpass characteristics of the GPR. The second step is concavity detection, which converts the filtered signal into binary-valued strings, where a ' 1 ' indicates a change in impedance of the target, and a ' 0 ' indicates no change in impedance.

After preprocessing, the crucial next step is to extract the characteristic mine strings from training data, such that the discrimination between mine and non-mine strings is maximized. Using the training data, the characteristic mine strings need to be found from a set of strings of relevant length from the different available 
landmine prototypes/exemplars. Towards this end, we propose a Markov chain model to characterize the statistics of the binary strings.

The state of the Markov model is defined as the number of successive zeros between the ones in the binary strings. Due to the inherent sparseness of the binarized GPR A-scans, this way of defining the states yields a smaller total number of states. We also quantize the states which makes the involved computation tractable. In order to identify the correct order of the model, we calculate the mean square difference (MSD) between the mine and non-mine string transition probabilities. This study reveals that Markov chain with order 1 or 2 is sufficient depending on the number of ones available in the strings considered.

The initial state and transition probabilities of the Markov chain allow us to compute the likelihood of each string. This information is useful to identify the best candidate mine strings yielding best detection and false alarm performance. We can also design a simple maximum likelihood-type detector which computes the likelihood of each A-scan given the mine string transitions. We present a few results showing the promise of such an approach.

In Section 2 we present the proposed Markov chain model for characterizing the binarized GPR A-scans. Section 3 presents results showing the effectiveness of the Markov model in capturing the statistics of the binary strings. The paper is concluded in Section 4 with a summary of the present work and future research directions.

\section{MARKOV CHAIN MODEL FOR BINARIZED GPR A-SCANS}

In this section, we develop a Markov chain model to characterize the statistics of the binary strings corresponding to mine and non-mine strings of GPR A-scans. We seek a computationally efficient model which captures the different transitions in the bit sequence of the strings in a statistical manner. We first define the states of the Markov chain, propose a quantization scheme to limit the number of total states, and finally discuss the usefulness of the model to compute string likelihood.

\subsection{Definition of Markov states}

Given a binary string of $L$ bits with $Z$ ' 1 's, one can define a two-state Markov chain model where the occurrence of ' 0 ' and ' 1 ' are the only two possible states. This is an over-simplified model because it can not adequately capture the correlation among the successive bits in the sequence. We choose to define the state in a manner which allows us to capture correlation among successive bits, exploits the sparseness inherent in the strings, and is computationally efficient.

We define a given Markov state as the number of zeros in between two consecutive ones such that the state $S_{i}$ indicates that there are $i$ successive zeros between the two ones under consideration. By this definition, the $L=12, Z=4$ bit string $S \triangleq 1 \underline{000} \underline{0100001}$ can be represented by the state sequence $S_{3} S_{1} S_{4}$. The state sequence $S_{3} S_{1} S_{4}$ means that the first ' 1 ' is followed by three zeros (represented by state $S_{3}$ ), which is followed by a ' 1 ', which is followed by a zero (represented by state $S_{1}$ ), which is followed by a ' 1 ', which is followed by four zeros (represented by state $S_{4}$ ), which is finally followed by the last ' 1 '. Due to the sparseness of the ' 1 's the same string can be represented by a sequence of 3 states, instead of 12 states required by the two-state model.

\subsection{Order of Markov chain}

With the above definition, we can calculate the initial state $(I)$ and transition probabilities $(P)$ of the Markov chain from the training data. In the considered GSTAMIDS (Ground Standoff Mine Detection System) GPR dataset, most of the mine and non-mine binary strings are of length $L=50$ to 100 , with number of ones $Z=3$ to 10. It was empirically found that for the vast majority of cases the highest number of consecutive zeros observed in the strings is 60 . That is, it is sufficient to have $S_{i}$ with $\max (i) \triangleq i_{\max }=61$, where the last state is a dump state which includes all cases greater than 60 consecutive zeros. In this case, the total number of possible states is 61 .

To accurately account for the correlation among successive bits it is important to identify the correct order of the Markov chain. Intuitively, the higher the Markov chain order, the more accurately the correlation of 
the bits is incorporated into the transition matrix. For example, if order $d=1$, then the string $S=S_{3} S_{1} S_{4}$ includes two state transitions: $S_{1} \mid S_{3}$ and $S_{4} \mid S_{1}$. For $d=1$, the initial state vector has size $i_{\max } \times 1$, and the state transition matrix has size $i_{\max } \times i_{\max }$. On the other hand, if $d=2$, then $S$ has only one state transition: $S_{4} \mid S_{3} S_{1}$. For this case, the initial state matrix has size $i_{\max } \times i_{\max }$, and the state transition matrix has size $i_{\max } \times i_{\max } \times i_{\max }$. In general, a Markov chain with order $d>3$ has $d$-dimensional initial state probability hypercube with $i_{\max }^{d}$ elements, and $(d+1)$-dimensional transition probability hypercube with $i_{\max }^{d+1}$ elements.

Given a range of allowable string lengths $L$ and the allowable number of ones $Z$, we can estimate the parameters, $I$, the initial state probabilities, and $P$, the transition probabilities, of the Markov chain for a specified order $d$. For estimating the initial state probabilities, the first state(s) of each valid string is counted by incrementing the corresponding component of $I$. In the end, all the components of $I$ are normalized to convert them into valid probabilities. For estimating the state transition probabilities, all the state transitions of each valid string are counted by incrementing the corresponding elements of $P$, followed by normalization to render valid probabilities. For example, in the example of $S=S_{3} S_{1} S_{4}$ with $d=1$, $I_{3} \leftarrow I_{3}+1, P_{3,1} \leftarrow P_{3,1}+1$, and $P_{1,4} \leftarrow P_{1,4}+1$. Note that the first index in the subscript of $P$ indicates the from state and the second index indicates the to state of the transition. If $d=2$, then $I_{3,1} \leftarrow I_{3,1}+1$, and $P_{3,1,4} \leftarrow P_{3,1,4}+1$.

\subsection{State quantization}

From the above discussion we notice that the size of $P$ increases exponentially as $i_{\max }^{d+1}$. For $d=4$ and $i_{\max }=$ 61 , the number of elements in $P$ grows very quickly indeed $\left(61^{4+1}=844,596,301\right)$. We propose a quantization of the states such that a set of consecutive states $\left(S_{i}, S_{i+1}, \cdots, S_{i+q-1}\right)$ is lumped into a single state $S_{i}^{(q)}$. For example, a quantization of $q=5$ means we have the quantized states $S_{1}^{(5)}=\left(S_{1}, S_{2}, S_{3}, S_{4}, S_{5}\right), S_{2}^{(5)}=$ $\left(S_{6}, S_{7}, S_{8}, S_{9}, S_{10}\right), \cdots, S_{12}^{(5)}=\left(S_{56}, S_{57}, S_{58}, S_{59}, S_{60}\right), S_{13}^{(5)}=S_{61}$. For $q=10$, the quantized states are $S_{1}^{(10)}=\left(S_{1}, \cdots, S_{10}\right), S_{2}^{(10)}=\left(S_{11}, \cdots, S_{20}\right), \cdots, S_{6}^{(10)}=\left(S_{51}, \cdots, S_{60}\right), S_{7}^{(10)}=S_{61}$. The baseline case of $q=1$ means no quantization is performed on the states. With quantization $q=5$ and $q=10, P$ has $13^{4+1}=371,293$ and $7^{4+1}=16,807$ elements, respectively, when $d=4$.

Aside from reducing computational complexity, state quantization serves another important purpose. Without any quantization, the state transition probabilities in $P$ become more and more sparse as we go to higher orders of the Markov chain. This may in turn render the computed likelihoods of many strings zero, simply because a particular transition did not occur even once in the observed data. Quantizing the states as mentioned above helps smooth out such cases. Another point to note is that the number of available transitions decreases as the Markov order is increased for a fixed number of strings. This may be an issue when higher order Markov models are considered with a limited number of strings available. So, comparison and use of different Markov orders should be performed in conjunction with an appropriate state quantization level, and a sufficiently large number of strings.

\subsection{Likelihood of strings}

The binarized GPR truth data can be separated into two categories: mine strings and non-mine strings. The mine strings are found by extracting a specified volumetric region around the true locations of known landmines using truth information. The remaining GPR data is considered to be non-mine strings. Due to the high sidelobe leakage of the impulse GPR used to acquire the data, we used a guard region around each mine to ensure that no mine strings were considered as non-mine strings.

Once the initial state and transition probabilities for these two types of data, mine and non-mine strings, have been estimated for a specified state quantization and Markov order $(q, d)$, it is straightforward to calculate the likelihood for any given string. We denote the initial state and transition probabilities for the two data types explicitly as $I^{(q, d, m)}, P^{(q, d, m)}, I^{(q, d, \bar{m})}$, and $P^{(q, d, \bar{m})}$, where the notations $m$ and $\bar{m}$ stand for mine and non-mine information, respectively.

The computation of string likelihood is best illustrated by examples. Again, let us consider our example string $S=S_{3} S_{1} S_{4}$. The likelihood of $S$ given all mine and non-mine string information is denoted by $L^{(q, d, m)}(S)$ and $L^{(q, d, \bar{m})}(S)$, respectively. Given all mine string information, we can compute the different 
string likelihoods for $q=1,2$ and $d=1,2$ as follows. The likelihoods when non-mine string information is given can be computed similarly.

$$
\begin{aligned}
& L^{(1,1, m)}\left(S_{3} S_{1} S_{4}\right) \equiv L^{(1,1, m)}\left(S_{3}^{(1)} S_{1}^{(1)} S_{4}^{(1)}\right)=I_{3}^{(1,1, m)} P_{3,1}^{(1,1, m)} P_{1,4}^{(1,1, m)} \\
& L^{(1,2, m)}\left(S_{3} S_{1} S_{4}\right) \equiv L^{(1,2, m)}\left(S_{3}^{(1)} S_{1}^{(1)} S_{4}^{(1)}\right)=I_{3,1}^{(1,2, m)} P_{3,1,4}^{(1,2, m)} \\
& L^{(2,1, m)}\left(S_{3} S_{1} S_{4}\right) \equiv L^{(2,1, m)}\left(S_{2}^{(2)} S_{1}^{(2)} S_{2}^{(2)}\right)=I_{2}^{(2,1, m)} P_{2,1}^{(2,1, m)} P_{1,2}^{(2,1, m)} \\
& L^{(2,2, m)}\left(S_{3} S_{1} S_{4}\right) \equiv L^{(2,2, m)}\left(S_{2}^{(2)} S_{1}^{(2)} S_{2}^{(2)}\right)=I_{2,1}^{(2,2, m)} P_{2,1,2}^{(2,2, m)} .
\end{aligned}
$$

String likelihoods computed using the Markov chain probabilities can be very useful to find the characteristic mine strings from training data. It can be used as a measure to identify which of the mine strings are more likely across the different landmine exemplars. Eventually, it may be possible to design landmine detectors that discriminate between landmine and clutter based on the likelihood of observed strings during training phase. In particular, it can be utilized as a feature to classify mine strings from non-mine strings. We will explore this aspect in detail in our future work.

It is interesting to note that once the Markov chain parameters have been estimated, it is possible to assign a probability to a newly encountered string not observed previously. Moreover, the Markov parameters, namely the initial state and transition probabilities, can be dynamically updated as new GPR data is gathered. This makes it possible to develop landmine detection schemes capable of dynamically adapting to different operating conditions due to soil, clutter and other environmental variations.

\section{RESULTS}

We developed and evaluated the Markov chain model using the GPR data collected at a government test site. The acquired amplitude GPR signal is converted into binary strings by inverse filtering followed by peak detection. ${ }^{6}$ The mine and non-mine strings are separated into two groups by using ground truth information. The initial state and transition probabilities are calculated as described in Section 2.

\subsection{MSD of mine and non-mine strings}

To determine the correct order of the Markov chain, we calculate the mean square difference (MSD) of the transition probabilities $P^{(q, d, m)}$ and $P^{(q, d, \bar{m})}$ for $Z=3,4, \cdots, 10$ and $d=1,2,3,4$. Since the dimensions of $P$ depend on the chosen $d$, we compute MSD by normalizing by the number of elements in $P$. The MSD for $(q, d)$ is computed as

$$
\operatorname{MSD}(q, d)=\frac{\sum_{i=1}^{i_{\max }} \sum_{j=1}^{i_{\max }} \cdots \sum_{l=1}^{i_{\max }}\left(P_{i, j, \cdots, l}^{(q, d, m)}-P_{i, j, \cdots, l}^{(q, d, \bar{m})}\right)^{2}}{i_{\max }^{d+1}},
$$

where $\{i, j, \cdots, l\}$ denotes the set of $(d+1)$ indices corresponding to the $(d+1)$ dimensions of $P$.

To find the correct order of the Markov chain, we identify which order maximizes the MSD between the mine and non-mine strings. In Fig. $1, \operatorname{MSD}(q, d)$ is plotted as a function of the number of ones $(Z)$ in the strings for $q=1\left(i_{\max }=61\right)$ and $d=1,2,3$. The MSD for $d=4$ is not calculated because of the large memory requirements for this case ( $P$ consists of $61^{5}$ elements). The same is plotted in Figs. 2 and 3 for $d=1,2,3,4$, using $q=5\left(i_{\max }=13\right)$ and $q=10\left(i_{\max }=7\right)$, respectively. For the different quantization levels, we notice that the MSD is maximized for $d=1,2$ depending on the value of $Z$. In particular, the MSD is maximized for $d=2$ when $Z=4$ and $Z=5$ for all quantization levels $q=1,5,10$. For rest of the values of $Z, d=1$ yields the highest MSD, with the exception of $Z=8$ for $q=10$ (see Fig. 3 ). 


\subsection{Likelihood of strings}

As described in Section 2, we can compute the likelihood of any string once the initial state and transition probabilities have been estimated. In Fig. 4, we plot the likelihood of all mine strings for both mine and non-mine state transition probabilities, $P^{(q, d, m)}$ and $P^{(q, d, \bar{m})}$. For the given GPR data set considered, there are 23,867 mine and 373,849 non-mine strings with $Z \in[3,20]$.

In Fig. 4 we plot the likelihood (in descending order, blue color) of all 23,867 mine strings computed using mine string transitions $P^{(q, d, m)}$ for $(q, d)=(5,1)$. On the same plot we also show the the likelihood of the same mine strings computed using non-mine string transitions $P^{(q, d, \bar{m})}$ for $(q, d)=(5,1)$ (red color). So this plot indicates whether particular mine strings are more likely or less likely to occur in mine exemplars/prototypes compared to non-mine regions. The fact that particular mine strings can be more likely to occur in non-mine regions compared to mine regions is due to the fact that the specification of the mine locations provided in truth information of the training data is not absolutely precise, and the specified mine regions can contain typical non-mine responses.

Clearly, Fig. 4 shows that only a small fraction of all the 23,867 mine strings is significantly highly likely to occur in the mine regions compared to non-mine regions. The best characteristic mine $\operatorname{string}(\mathrm{s})$ with attractive detection performance should be chosen from this subset of mine strings. Fig. 5 shows a similar plot as Fig. 4, but for $Z=6$ only, which includes 5,123 mine strings.

\subsection{Mine detection based on likelihood of strings}

To test whether the proposed Markov model and the associated string likelihood computation is useful in detecting actual mines, we computed likelihoods of strings of a brick (512 deep x 51 crossrange x 100 downrange) of actual GPR data comprising an area of approximately $12.75 \mathrm{~m}^{2}$. The string likelihoods are computed using $P^{(q, d, m)}$ for $(q, d)=(5,1), L \in[50,100]$ and $Z \in[6,10]$. For each A-scan in the brick, multiple strings may exist within the specified range of string length and the number of ' 1 's, $L$ and $Z$. In such a case only the maximum of all the different string likelihoods of a given A-scan is retained.

Figs. 6,7, and 8 show six such example bricks. In Figs. 6 and 7, the string likelihoods converge at the locations of true mines within the brick. However, in Fig. 8, the string likelihoods do not converge at the true mine locations, instead sporadic hotspots are observed across the bricks. This is an encouraging result since it shows that it may be possible to design mine detectors based on string likelihoods derived from a Markov chain model of binarized GPR signals. Of course, the feasibility of detector design based on the proposed statistical string statistics requires further study.

\section{CONCLUSION AND FUTURE DIRECTIONS}

We proposed a Markov chain model to characterize the string statistics of binarized GPR signals for syntactic landmine detection. An efficient and computationally tractable definition of the Markov states were provided. The order of the Markov chain was determined empirically by observing which order maximizes the mean square difference between mine and non-mine strings. The Markov parameters, namely, the initial state and transition probabilities, were estimated and used to compute the likelihood of any given string. Finally, the likelihood of strings was shown to be an effective measure for discriminating mine and non-mine strings.

The proposed Markov model only dealt with strings and their state transitions in the depth direction. However, it is possible to generalize this model to include transitions in the cross range and down range directions as well. This would allow the model to capture more information regarding the mine, thereby allowing a more accurate characterization of the landmine string statistics. Another way to include more information is to work with ternary strings rather than binary strings by distinguishing between positive and negative peaks of the preprocessed GPR signals. Yet another interesting avenue of research may be to explore hidden Markov processes for modeling the binary/ternary strings. This may be a powerful tool since it may allow us to estimate the true unobserved impedance discontinuity profile of the target in an effective manner. 


\section{ACKNOWLEDGMENTS}

The authors would like to thank the Office of Naval Research for supporting this work under Grant No. N00014-08-1-0629. The authors would also like to express their gratitude to the Night Vision and Electronic Sensors Directorate (NVESD), Ft. Belvoir, VA, for providing sensor data.

\section{REFERENCES}

[1] D. J. Daniels, "A review of landmine detection using GPR," Proc. European Radar Conf. (EuRAD'08), pp. 280-283, Oct. 2008.

[2] P. A. Gader, M. Mystkowski, and Y. Zhao, "An abrupt change detection algorithm for buried landmines localization," IEEE Trans. Geosci. Remote Sens. 44, pp. 260-272, Feb. 2006.

[3] J. N. Wilson, P. Gader, L. Wen-Hsiung, H. Frigui, and K. C. Ho, "A Large-Scale Systematic Evaluation of Algorithms Using Ground-Penetrating Radar for Landmine Detection and Discrimination," IEEE Trans. Geosci. Remote Sens. 45, pp. 2560-2572, Aug. 2007.

[4] H. Frigui, L. Zhang, and P. Gader, "Context-dependent multisensor fusion and its application to land mine detection," IEEE Trans. Geosci. Remote Sens. 48, pp. 2528-2543, June 2010.

[5] K. J. Hintz, N. Peixoto, and D. Hwang, "Syntactic landmine detection and classification," in Detection and Sensing of Mines, Explosive Objects, and Obscured Targets XIV, R. S. Harmon, J. T. Broach, J. H. Holloway, Jr., ed., Proc. SPIE 7303, May 2009.

[6] A. O. Nasif, K. J. Hintz, and N. Peixoto, "Preprocessing of GPR data for syntactic landmine detection and classification," in Detection and Sensing of Mines, Explosive Objects, and Obscured Targets XIV, R. S. Harmon, J. T. Broach, J. H. Holloway, Jr., ed., Proc. SPIE 7664, Apr. 2010. 


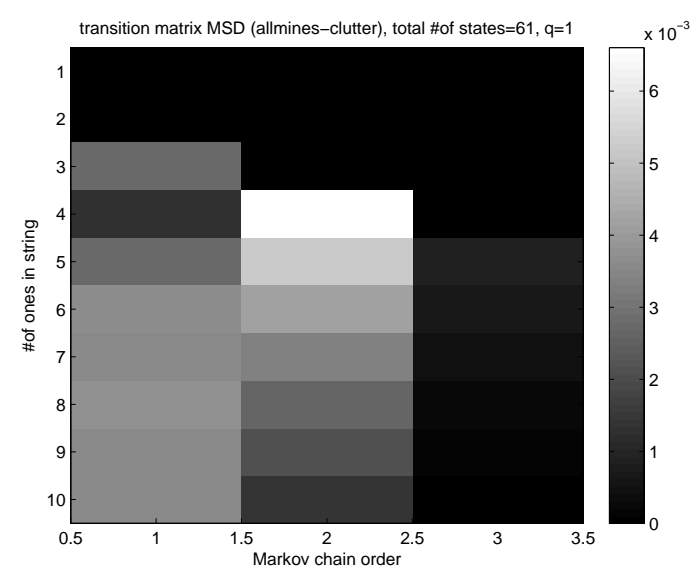

Figure 1: $\operatorname{MSD}(q, d)$ for $q=1\left(i_{\max }=61\right)$ as a function of $Z$ (the number of ' 1 's) for Markov chain order $d=1,2,3$.

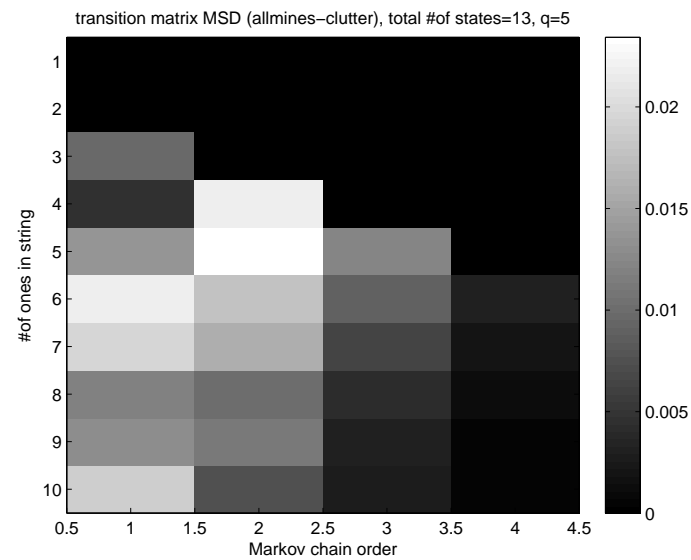

Figure 2: $\operatorname{MSD}(q, d)$ for $q=5\left(i_{\max }=13\right)$ as a function of $Z$ (the number of ' 1 's) for Markov chain order $d=1,2,3,4$

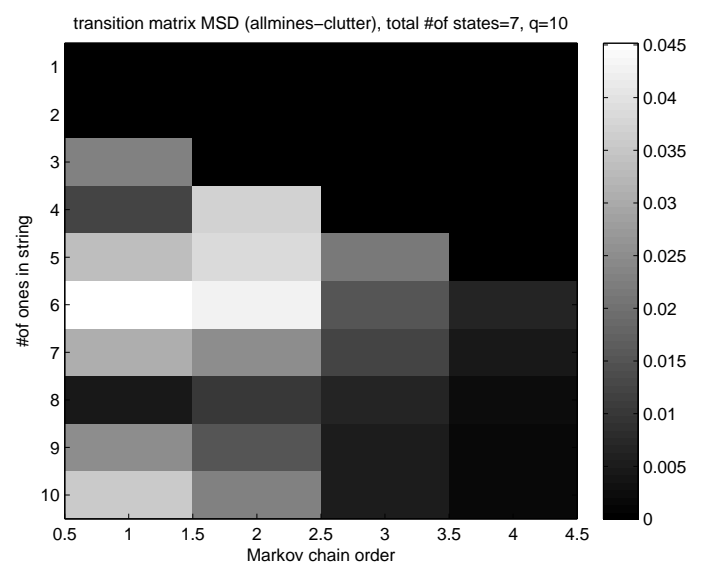

Figure 3: $\operatorname{MSD}(q, d)$ for $q=10\left(i_{\max }=7\right)$ as a function of $Z$ (the number of ' 1 's) for Markov chain order $d=1,2,3,4$. 


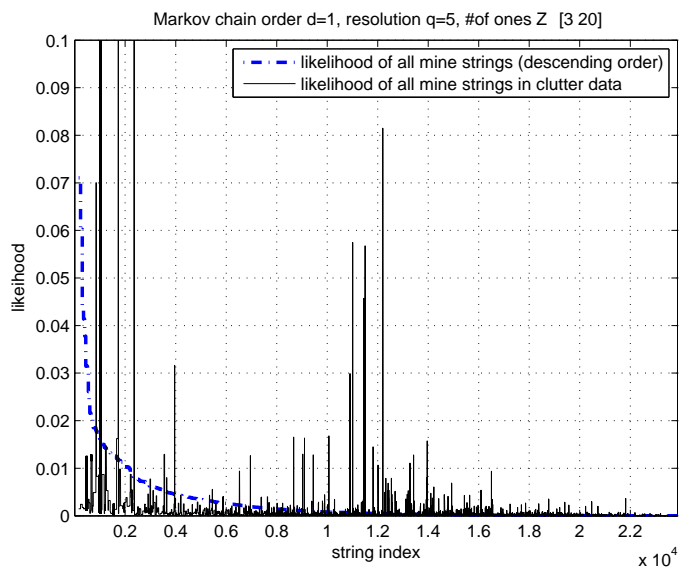

Figure 4: Likelihood of mine strings given mine string transition probabilities $P^{(q, d, m)}$ and non-mine string transition probabilities $P^{(q, d, \bar{m})}$ for $(q, d)=(5,1)$ and $Z \in[3,10]$.

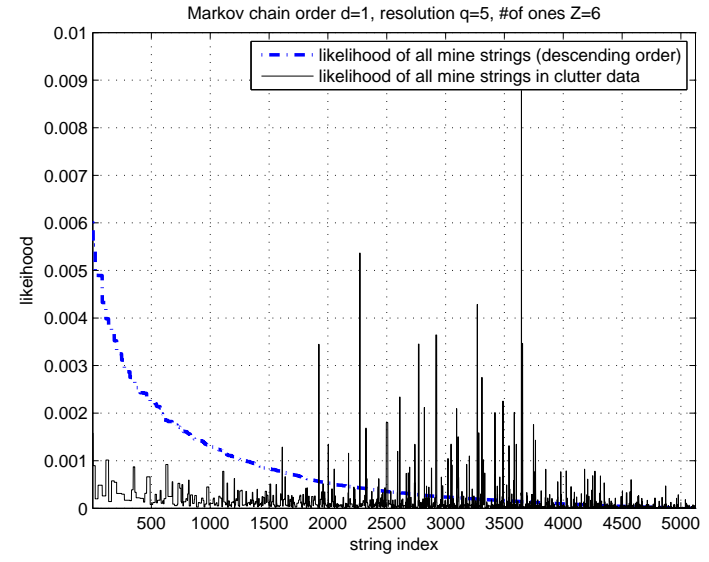

Figure 5: Likelihood of mine strings given mine string transition probabilities $P^{(q, d, m)}$ and non-mine string transition probabilities $P^{(q, d, \bar{m})}$ for $(q, d)=(5,1)$ and $Z=6$.
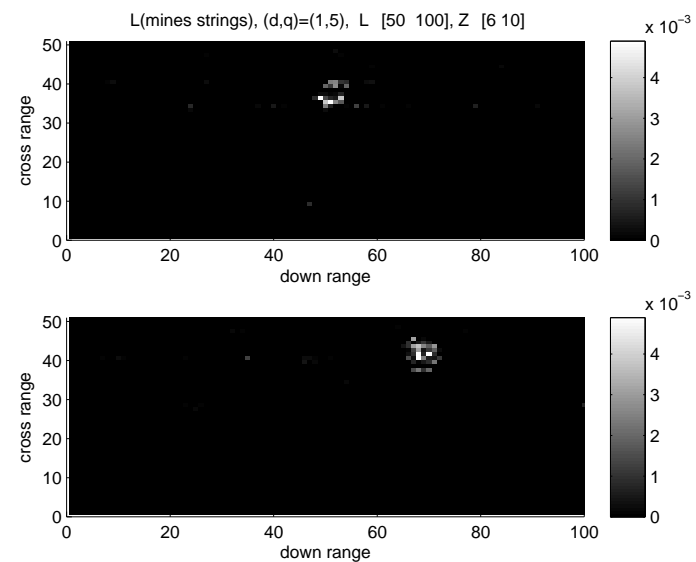

Figure 6: Projected maximum string likelihood of two bricks (each of approximately $12.75 \mathrm{~m}^{2}$ region) of actual GPR data. The string likelihoods are computed using $P^{(q, d, m)}$ for $(q, d)=(5,1)$ and $Z \in[6,10]$. For this example the likelihood of the computed strings converge around the true mine locations. 


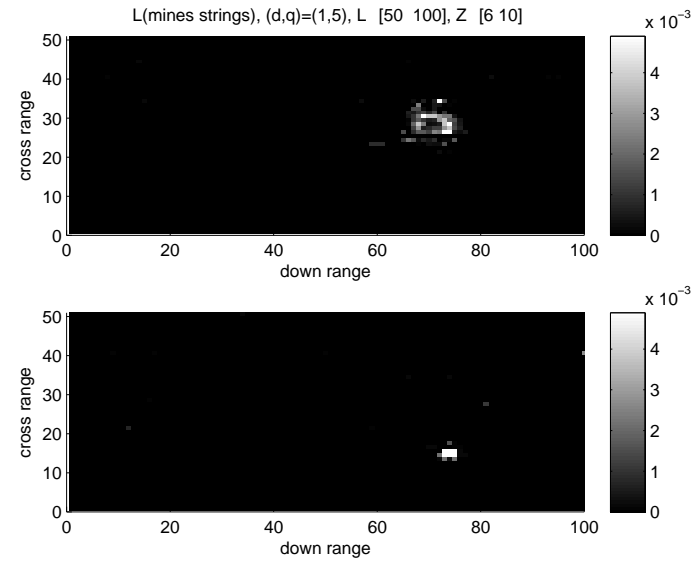

Figure 7: Projected maximum string likelihood of two bricks (each of approximately $12.75 \mathrm{~m}^{2}$ region) of actual GPR data. The string likelihoods are computed using $P^{(q, d, m)}$ for $(q, d)=(5,1)$ and $Z \in[6,10]$. For this example the likelihood of the computed strings converge around the true mine locations.

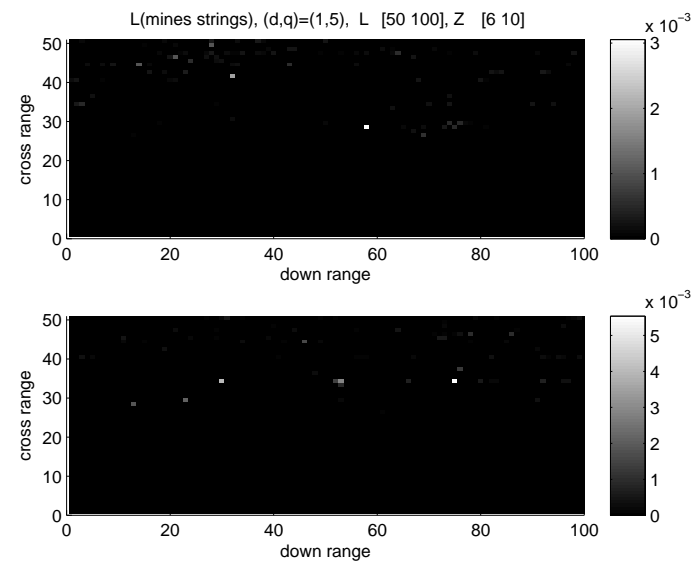

Figure 8: Projected maximum string likelihood of two bricks (each of approximately $12.75 \mathrm{~m}^{2}$ region) of actual GPR data. The string likelihoods are computed using $P^{(q, d, m)}$ for $(q, d)=(5,1)$ and $Z \in[6,10]$. For this example the likelihood of the computed strings do not converge around the true mine locations as in the cases of Figs. 6 and 7 . 